\title{
Topical Phytotherapy is the Nobel Approach for Revision of Total Knee Replacement: A Unique Case Study
}

\begin{abstract}
Knee-osteoarthritis (KOA) can only be treated by total knee replacement (TKR), which is a well-known surgical intervention in the globe. But in some cases, TKR failed in the operated knee-joint as well as non-operated knee-joint may pose risk of KOA. In general, patients are advised to undergo revision of TKR when two prostheses of femora and tibial compartments a re prolapsed or loosen or such other rea sons as well as non-operating knee-joint needs to be operated urgently. The phytotherapy with Indian medicinal plants are well-established. The present case study was aimed to determine the efficacy of topical phytotherapeutic treatment for 90 days in order to prevent the revision of TKR, improve the pain and functional performance in daily activities in four Indian patients. After 90 days of topical phytotherapy treatment, the results were observed a significant decrease in pain in both the legs and lower back under visual analogue scale $(p \varangle 0.05)$, improvement of pain $(p<0.05)$, stiffness $(p<0.05)$ and physic al function $(\mathrm{p}<0.05)$ under WOMAC Index, a significant improvement in the Karnofsky scale $(<0.05)$, reduction of obesity confirmed by body mass index $(p<0.05)$ and nomal radiological features. In conclusion, failed TKR c a n be treated by phytothera py without further revision evidenced by nomalization of above-mentioned parameters.
\end{abstract}

Keywords: Knee osteoarthritis; Total knee replacement; Topical phytotherapy; Revision of knee-joints by phytotherapy

\section{Introduction}

Total knee replacement (TKR) is a common and ultimate treatment of knee osteoarthritis (KOA) in India and other parts of the globe. Basically, the concept of TKR is mainly to relief pain and improvement of performance in daily activities $[1,2]$. However, TKR is done on the basis of different prostheses design such as cruciate retaining, posterior stabilized and high flexion posterior stabilized prostheses [2-6]. According to Julin J et al. there is a risk of prosthesis failure in young age groups of $<55$ years old due to infection, obesity, physical activity, loosening, osteolysis, etc. and needs revision for further prosthesis [7-9]. In an earlier study, surgical outcome revealed dissatisfaction in patients [10]. In the present report, it was aimed to detect efficacy of topical phytotherapeutic treatment for preventing the revision of TKR with normalization of daily functional activities without pain, reduction of body weights and also improved the damaged knee-joints in four Indian patients.

\section{Case Study}

Four female patients aged $65,73,55$ and 64 years old respectively advised to further revision of unilateral TKR because they suffered with severe pain on weight-bearing, impaired function, and limitations of daily activities. They were treated at OPTM Healthcare (P) Ltd., India, from January 2016 to July 2016. The study protocol was evaluated and approved by the OPTM Research Institute Ethics Committee. An institutional review board-approved consent form for physical examinations and bilateral knee-joint images (X-rays) required for the study was signed by all four patients. Baseline demographic characteristics of four patients are shown in Table 1. The said four

\section{Journal of}

Orthopedics \& Rheumatology

\author{
Apurba Ganguly* \\ Founder and Head Researcher, OPTM Research Institute, India \\ *Address for Correspondence \\ Apurba Ganguly, Founder and Head Researcher, OPTM Research \\ Institute, 145 Rashbehari Avenue, Kolkata - 700029, India, Tel: \\ +919830389616; Email: apurbaganguly15@gmail.com \\ Submission: 12 March, 2018 \\ Accepted: 25 April, 2018 \\ Published: 04 May, 2018 \\ Copyright: ๑ 2018 Ganguly A. This is an open access article distributed \\ under the Creative Commons Attribution License, which permits \\ unrestricted use, distribution, and reproduction in any medium, provided \\ the original work is properly cited.
}

patients were not being treated by oral medications; injections; massage with any type of herbal gels; and any type of alternative interventions or treatments for diminishing pain or inflammation, for muscle relaxation, or to improve of the skeletal muscles during the last four weeks prior to the treatment of 90 days. The radiological features were observed bone erosion and revision for further prosthesis needed in the post-operated knee joint and required surgical intervention in the non-surgical knee joint as the patients were suffering with severe pain, stiffness and physical functional disabilities in both the kneejoints (Table 1) (Figures 1A,1B, 2A,2B,3A,3B,4A and 4B). Four pair of radiological images (before and after) is depicted in Figures 1-4.

Different types of prostheses in the tibia compartment have been used during TKR in order to get successful results but in the present report of the four patients have shown the further revision of TKR required and have risk of secondary knee osteoarthritis and nonoperated knee-joint needs surgical intervention as shown in Figures $1 \mathrm{~A}, 1 \mathrm{~B}, 2 \mathrm{~A}, 2 \mathrm{~B}, 3 \mathrm{~A}, 3 \mathrm{~B}, 4 \mathrm{~A}$ and $4 \mathrm{~B}$. All the four patients decided not to go for surgery. Hence, author has taken as challenge to recover them with the help of topical phytotherapeutic treatment protocol.

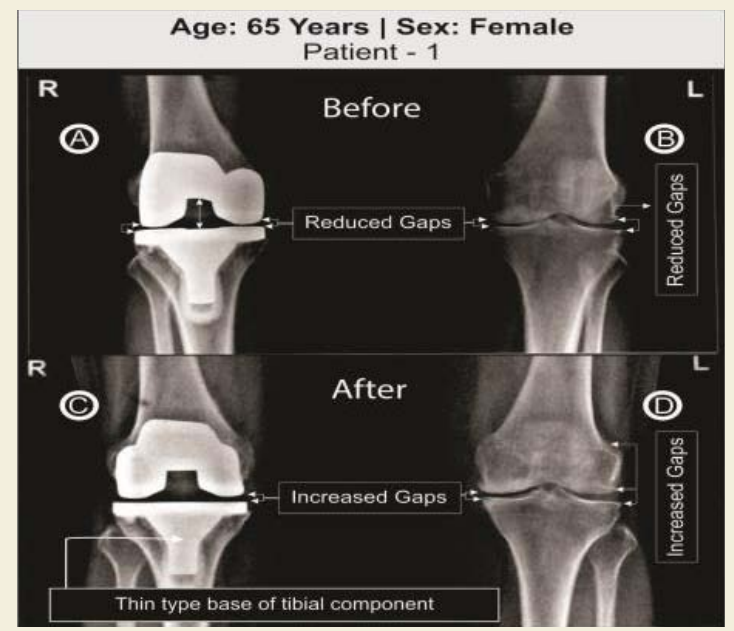

Figure 1: Radiological images for before and after the treatment. 
Citation: Ganguly A. Topical Phytotherapy is the Nobel Approach for Revision of Total Knee Replacement: A Unique Case Study J Orthopedics Rheumatol. 2018; $5(1): 6$.

Table 1: Demographic data and baseline characteristics of patients.

\begin{tabular}{|c|c|c|c|c|}
\hline Parameters & Patient-1 & Patient-2 & Patient-3 & Patient-4 \\
\hline Age (years) & 65 & 73 & 55 & 64 \\
\hline Gender & Female & Female & Female & Female \\
\hline Under gone TKR (right or left knee) & Right knee & Left knee & Left knee & Right knee \\
\hline TKR done at the age & 62 & 70.5 & 53 & 62.5 \\
\hline Weight (kg) & 87.94 & 82.13 & 76.94 & 70.6 \\
\hline BMI (kg/m2) & 32.3 & 32.9 & 33.3 & 31.8 \\
\hline Indian ethnic group & Bengali & Punjabi & Marathi & Gujrati \\
\hline Dietary habit & Non-vegetarian & Non-vegetarian & Vegetarian & Vegetarian \\
\hline Work status & Self employed & Housewife & Employed full time & Retired \\
\hline Marital status & Single & Married & Widow & Separated \\
\hline \multicolumn{5}{|c|}{ Classification of KOA (K-L system) } \\
\hline Left knee & $\geq$ Grade 4 & $\begin{array}{c}\text { Completely narrowed } \\
\text { the gap between the } \\
\text { prostheses }\end{array}$ & Prolapsed prostheses & $\geq$ Grade 4 \\
\hline \multicolumn{5}{|c|}{ Pain under VAS (mm) } \\
\hline Right leg & 45 & 90 & 89 & 89 \\
\hline Left leg & 98 & 55 & 56 & 53 \\
\hline Lower back & 61 & 42 & 62 & 65 \\
\hline \multicolumn{5}{|c|}{ Under WOMAC Index (\%) } \\
\hline Pain & 19.75 & 18.75 & 19.79 & 20.83 \\
\hline Stiffness & 7.29 & 4.17 & 6.25 & 8.33 \\
\hline Physical function & 68.75 & 66.67 & 67.7 & 68.75 \\
\hline \multicolumn{5}{|c|}{ Multiple complaints } \\
\hline Constipation & Present & Present & Not present & Present \\
\hline Acidity \& reflux & Not present & Present & Not present & Present \\
\hline urinery incontinence & Present & Not present & Present & Not present \\
\hline Crepitus during knee flxion & Present & Present & Present & Present \\
\hline Morning stiffness (<30 min) & Present & Present & Present & Present \\
\hline \multicolumn{5}{|c|}{ Measures taken to diminished pain \& inflammation } \\
\hline Knee-cap (right or left) & Bilateral & Right knee & Bilateral & Bilateral \\
\hline Paracetamal and NSAIDs & Paracetamol & NSAIDs & NSAIDs & Paracetamol and NSAIDs \\
\hline Corticosterial injection & 6 times in 6 months & 2 times in 2 months ago & 8 times in 4 months & 12 times in 3 months \\
\hline Walking support & Stick & Wheel chair & Sketches & A walker \\
\hline Physiotherapy done(years) & 3 & 2.5 & 2 & 1.5 \\
\hline Undergoing alternative treatment & Homeopathic & Ayurvedic \& gels & Acupuncture & Ayurvedic\& Homeopathic \\
\hline $\begin{array}{l}\text { Supplements taken to reduce pain } \\
\text { improve fitness }\end{array}$ & Calcium \& vitamin D & Glucosumine & Calcium \&vitamin D & Glocosumine\& Chondroitin \\
\hline
\end{tabular}

The main objectives of the treatment are: to reduce pain, inflammation and stiffness of muscles without dependence upon any types of pain killers or corticosteroid injection or arthrocentesis; to increase the muscular strength without using supporting belt on the waist or knee joints and the gaps between the bones/ vertebrae; to rectify calcifications/degeneration of bones, and to normalize the varus/valgus deformities (Figures 1-4) [10-12].

The treatment involves topical application of phytoconstituents from the extracts of six Indian medicinal plants namely Cissus quadrangularis (whole plant), Heliotropium indicum (whole plant), Rosemarinus officinalis (leaves and flowers), Calotropis gigantea (root and leaves), Boswellia serratia (resin) and Curcuma longa (rhizome) mixed with virgin sesame oil (extracted from seeds at room temperature) and beehives wax to make viscous phyto-based oil without using any preservatives or chemicals in order to preserve the phytochemical properties of plants intact. The virgin sesame oil is acted as bio-preservative and beehive wax helps to reduce joint pain, to relieve stiffness, to stimulate circulation and to moisturize skin [11-18].

Several researchers had already reported the medical effects (specially on pain, inflammation and stiffness of muscles) on human body of the phytochemicals contained in Cissus quadrangularies, Heliotropium indicum, Rosemarinus officinals, Calotropis gigantean, Boswellia serrata and Curcuma longa [14-16,19-23].

Each $30 \mathrm{ml}$ of said viscous phyto-based oil is to be applied with the 
Citation: Ganguly A. Topical Phytotherapy is the Nobel Approach for Revision of Total Knee Replacement: A Unique Case Study J Orthopedics Rheumatol. 2018; $5(1): 6$.

tip of three fingers in particular technique over the skin three times a day with minimum interval of time two hours for 90 days; lying in six different postural positions such as supine, prone, right and left contralateral and right and left cross (supper) contralateral in different programmed sequences in order to nourished the effected group of badly damaged muscles and nerves in the legs and lumber regions during KOA $[11,13,15,24]$. The author had previously discussed

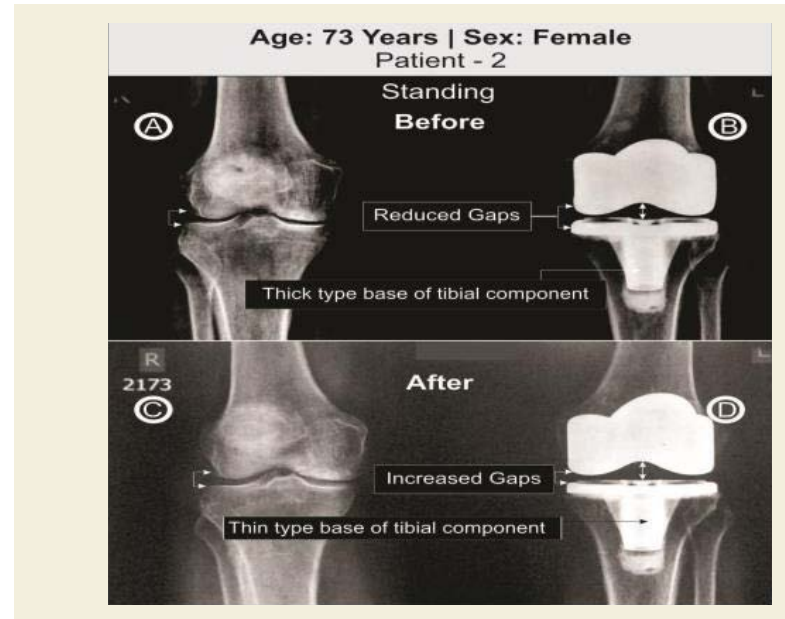

Figure 2: Radiological images for before and after the treatment.

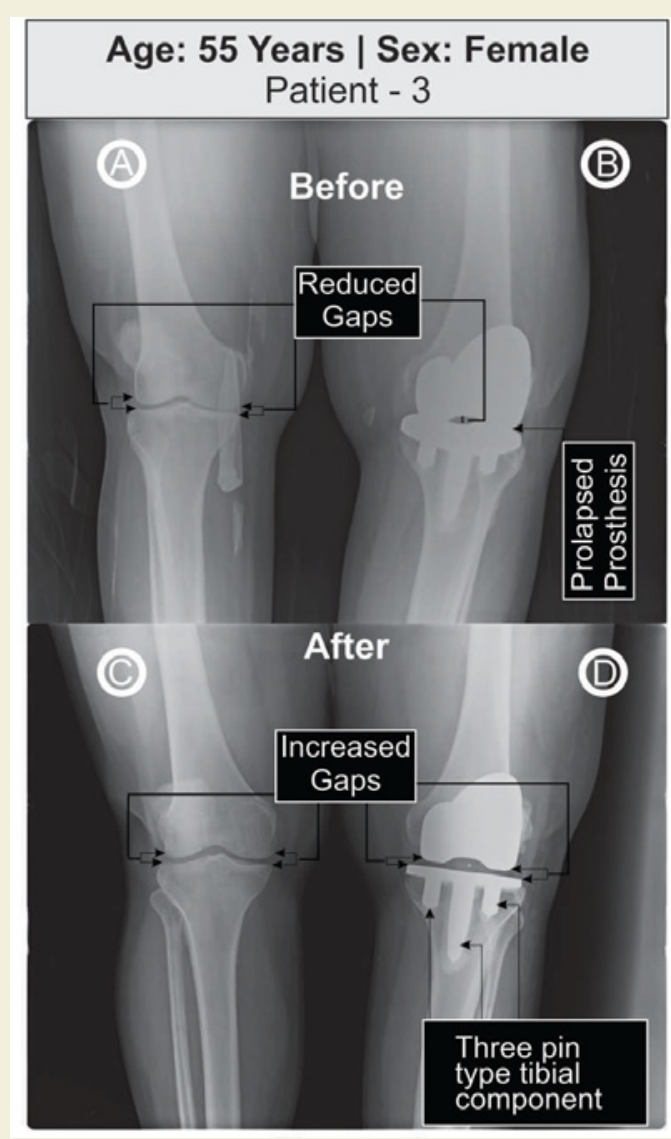

Figure 3: Radiological images for before and after the treatment.

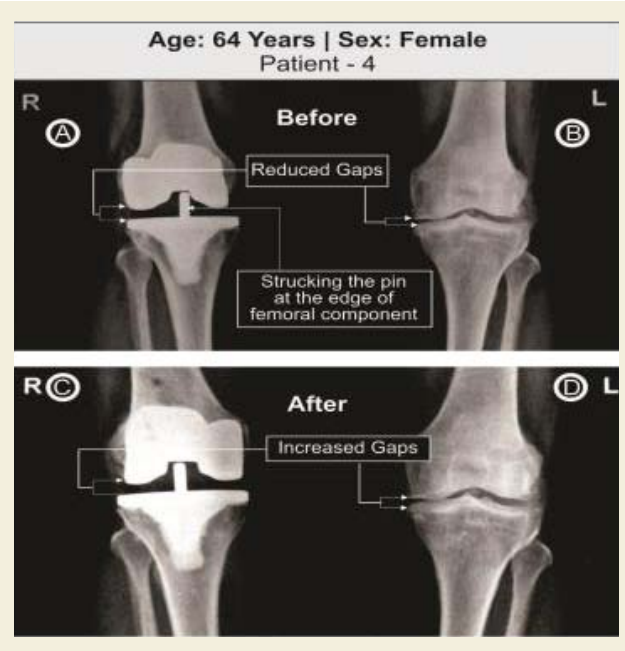

Figure 4: Radiological images for before and after the treatment.

elaborately, the reasons of the programmed sequences with six postural positions, and the purpose of special devices such as medicated pad, wooden roller and bet tarry operated electronic nerve stimulator used during the therapy $[11,13,24]$.

The treatment protocol is based on well defined certain principles and theories and also based on the fundamental properties of all muscles such as excitability, conductivity, contractibility, elasticity and viscosity with the help of well known chemical, mechanical, thermal and electrical stimuli $[11,13,24]$. In the phytotherapy protocol, viscous phyto-based oil serves the purpose of producing chemical stimulation, manipulation with the tip of three fingers and Wooden roller develop mechanical stimulation, medicated pads with control temperature generates thermal stimulation and 9 volt DC electronic nerve stimulator produces electrical stimulation $[11,24,25]$.

Tables 2 and 3, summarize the pain and performance parameters of four patients. Patients underwent standing X-ray examination to the most important leg joints and lower back: it confirmed knee involvement with large osteophytes, narrowing the joint space, increased density of the subchondral bone, large subchondral cysts in the non-operated knee joints. The joint spaces of the operated knee joint were completely jointed in case of patients 1 and 3, substantially diminished in case of patient 2 and stucking the long fixed pin at the

Table 2: Baseline characteristics of pain for four patients.

\begin{tabular}{|c|c|c|c|c|}
\hline \multicolumn{5}{|c|}{ VAS (in mm) } \\
\hline Right Leg & 45 & 90 & 89 & 89 \\
\hline Left Leg & 98 & 55 & 56 & 53 \\
\hline Lower back & 61 & 42 & 62 & 65 \\
\hline \multicolumn{5}{|c|}{ WOMAC Index (\%) } \\
\hline Pain & 19.75 & 18.75 & 19.79 & 20.83 \\
\hline Stiffness & 7.29 & 4.14 & 6.25 & 8.33 \\
\hline Physical function & 68.75 & 66.67 & 67.7 & 68.75 \\
\hline KPS (\%) & 40 & 50 & 40 & 30 \\
\hline
\end{tabular}

VAS: Visual Analogue Scale; WOMAC Index: The Western Ontario and McMaster Universities Osteoarthritis Index; KPS: Karnofsky Performance Score 
Citation: Ganguly A. Topical Phytotherapy is the Nobel Approach for Revision of Total Knee Replacement: A Unique Case Study J Orthopedics Rheumatol. 2018; $5(1): 6$.

ISSN: $2334-2846$

Table 3: Analysis of VAS, WOMAC Index, KPS and BMI for four patients.

\begin{tabular}{|c|c|c|c|c|c|c|}
\hline & \multirow{3}{*}{\begin{tabular}{|c|} 
Baseline \\
$\begin{array}{c}\text { Mean } \\
\text { (SEM) }\end{array}$ \\
\end{tabular}} & \multirow{3}{*}{$\begin{array}{c}\text { After end of } \\
90 \text { days } \\
\text { Mean (SEM) }\end{array}$} & \multicolumn{4}{|c|}{ Improvement after 90 days } \\
\hline & & & \multirow[b]{2}{*}{ MD } & \multicolumn{2}{|c|}{$95 \% \mathrm{Cl}$} & \multirow[b]{2}{*}{ p-value } \\
\hline & & & & $\begin{array}{l}\text { Lower } \\
\text { Limit }\end{array}$ & $\begin{array}{l}\text { Upper } \\
\text { Limit }\end{array}$ & \\
\hline \multicolumn{7}{|c|}{ VAS (mm) } \\
\hline Right leg & $\begin{array}{c}78.2 \\
(11.08)\end{array}$ & $5.00(0.41)$ & 73.2 & 46.06 & 100.34 & 0.0006 \\
\hline Left leg & $\begin{array}{c}65.50 \\
(10.85)\end{array}$ & $3.50(0.29)$ & 62 & 35.44 & 88.56 & 0.0012 \\
\hline Lower back & $\begin{array}{l}57.50 \\
(5.22)\end{array}$ & $2.50(0.29)$ & 55 & 42.21 & 67.79 & $<0.0001$ \\
\hline \multicolumn{7}{|c|}{ WOMAC INDEX (\%) } \\
\hline Pain & $\begin{array}{l}19.78 \\
(0.42)\end{array}$ & $1.56(0.30)$ & 18.22 & 16.95 & 19.45 & $<0.0001$ \\
\hline Stiffness & $\begin{array}{c}6.50 \\
(0.89)\end{array}$ & $0.00(0.00)$ & 6.5 & 4.31 & 8.69 & 0.0003 \\
\hline $\begin{array}{l}\text { Physical } \\
\text { function }\end{array}$ & $\begin{array}{l}67.97 \\
(0.49)\end{array}$ & $11.98(0.49)$ & 55.99 & 54.27 & 57.7 & $<0.0001$ \\
\hline KPS (\%) & $\begin{array}{l}40.00 \\
(4.08)\end{array}$ & $70(4.08)$ & -30 & -44.12 & -15.88 & 0.002 \\
\hline $\operatorname{BMI}\left(\mathrm{Kg} / \mathrm{m}^{2}\right)$ & $\begin{array}{l}32.57 \\
(0.33)\end{array}$ & $28.24(0.40)$ & 4.33 & 3.05 & 5.61 & 0.0002 \\
\hline
\end{tabular}

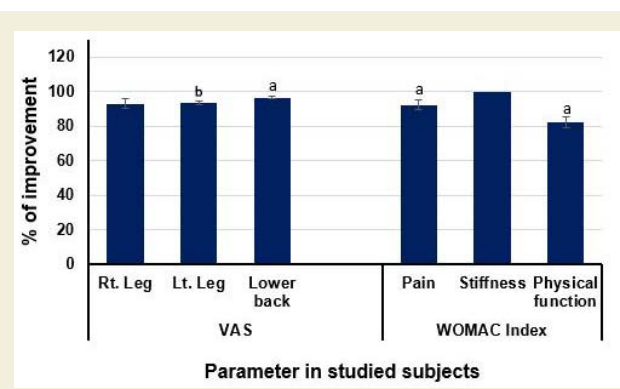

Figure 5: Percentage improvement of parameters under VAS and WOMAC Index of four patients who had undergone TKR $\left({ }^{\mathrm{a}} \mathrm{P}<0.001\right.$; $\left.{ }^{\mathrm{b}} \mathrm{P}<0.05\right)$.

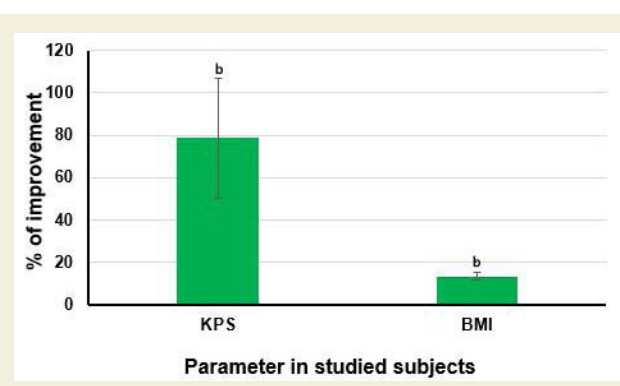

Figure 6: Percentage improvement under Karnofsky performance scale and body mass index of four patients who had undergone TKR $\left({ }^{(} \mathrm{P}<0.05\right)$.

corner of the femoral compartment in case of patient 4 (Figures 1-4).

After 90 days of topical phytotherapeutic treatment, improvements were observed in pain under visual analogue scale (VAS) and pain, stiffness and physical function under WOMAC Index with highly significant values $(\mathrm{p}<0.05)$; improvements of functional performance under the Karnofsky performance scale (KPS) and reduction of obesity as confirmed by body mass index (BMI) were also highly statistically significant $(\mathrm{p}<0.05)$ (Table 3) (Figures 5 and 6). A substantial improvement of the knee-joints (both non-operated and operated) have also been observed as evidenced by $\mathrm{X}$-ray imaging (Figure 1C,1D,2C,2D,3C,3D,4C and 4D).

\section{Discussion}

The present case reports indicate the failure in TKR and osteoporosis after TKR with pain and non-performance of daily activities for which alternative treatment with specialised topical phytotherapy for 90 days would be considered to be best. Generally, the TKR supports to relief pain and improvement of performance in daily activities $[1,2]$. Further revision on TKR may be stressful, risk and expensive but specialized topical phytotherapeutic treatment after 90 days to the studied patients are suitable therapy where substantial improvement in pain, stiffness, functional and performance abilities, BMI with highly statistically significant values $(\mathrm{p}<0.05)$, knee gaps, osteoporosis and osteophytes in non-operated knee-joint and knee flexion were observed (Table 3) (Figures 1C,1D,2C,2D,3C,3D,4C,4D, 5 and 6).

\section{Conclusion}

The aim of the TKR is to reduce the pain, increase the functional ability and the gap between the femoral and tibial compartments. The present study firmly confirmed with normalization of prolapsed prostheses by increasing the gap between the compartments of the operated and non-operated knee-joints as evidenced by $\mathrm{X}$-ray images and diminishing pain, stiffness and improvement of physical functional and performance abilities and reduction of inflammation as well as obesity confirmed by BMI with the help of topical phytotherapeutic method within 90 days (Table 3) (Figures 1C,1D,2C,2D,3C,3D,4C,4D,5 and 6).

Further researches should be undertaken on:

1. Deranged anatomical and abnormal biochemical features after TKR,

2. Phytochemicals characterization by using Mass Spectroscopy,

3. Measurements of collagen and 4-hydroxyplroline (O-Hyp) to show collagen formation, which takes place with the treatment, and

4. Measurements of hyaluronic acid both in volume and chemical compositions before and after the treatment to detect how the phytochemicals changing the chemical compositions of hyaluronic acid.

\section{References}

1. Hepinstall MS, Ranawat AS, Ranawat CS (2010) High-flexion total knee replacement: functional outcome at one year. HSS J 6: 138-144.

2. Lee SM, Seong SC, Lee S, Choi WC, Lee MC (2012) Outcomes of the different types of total knee arthroplasty with the identical femoral geometry. Knee Surg Relat Res 24: 214-220.

3. Ranawat CS, Luessenhop CP, Rodriguez JA (1997) The press-fit condylar modular total knee system. Four-to-six-year results with a posterior-cruciatesubstituting design. J Bone Joint Surg Am 79: 342-348.

4. Buehler KO, Venn-Watson E, D'Lima DD, Colwell CW Jr (2000) The press-fit condylar total knee system: 8- to 10-year results with a posterior cruciateretaining design. J Arthroplasty 15: 698-701.

5. Kim YH, Sohn KS, Kim JS (2005) Range of motion of standard and high- 
Citation: Ganguly A. Topical Phytotherapy is the Nobel Approach for Revision of Total Knee Replacement: A Unique Case Study J Orthopedics Rheumatol. 2018; $5(1): 6$.

flexion posterior stabilized total knee prostheses. A prospective, randomized study. J Bone Joint Surg Am 87: 1470-1475.

6. Rasquinha VJ, Ranawat CS, Cervieri CL, Rodriguez JA (2006) The pressfit condylar modular total knee system with a posterior cruciate-substituting design. A concise follow-up of a previous report. J Bone Joint Surg Am 88: 1006-1010.

7. Julin J, Jämsen E, Puolakka T, Konttinen YT, Moilanen T (2010) Younger age increases the risk of early prosthesis failure following primary total knee replacement for osteoarthritis. A follow-up study of 32,019 total knee replacements in the Finnish Arthroplasty Register. Acta Orthop 81: 413-419.

8. Kim TK, Chang CB, Kang YG, Kim SJ, Seong SC (2009) Causes and predictors of patient's dissatisfaction after uncomplicated total knee arthroplasty. J Arthroplasty 24: 263-271.

9. Kim KT, Lee S, Ko DO, Seo BS, Jung WS, et al. (2014) Causes of failure after total knee arthroplasty in osteoarthritis patients 55 years of age or younger. Knee Surg Relat Res 26: 13-19.

10. Leta TH, Lygre SH, Skredderstuen A, Hallan G, Gjertsen JE, et al. (2016) Outcomes of unicompartmental knee arthroplasty after aseptic revision to total knee arthroplasty: a comparative study of 768 TKAs and 578 UKAs revised to TKAs from the Norwegian Arthroplasty Register (1994 to 2011). J Bone Joint Surg Am 98: 431-440.

11. Ganguly A (2015) Tropical phytotherapeutic treatment for achieving knee symmetry in osteoarthritis - A sustainable approach. Int J Phytomedicine 6: 489-509.

12. Ganguly A (2016) Normalization of varus/valgus deformities in osteoarthritis by exteral application of phytoconstituents: confirmed with anatomical observations and biochemical profiles and radiological images. Anat Physiol 6: 224.

13. Ganguly A (2015) Obtaining normal flexion and extension of knee joints on supine, prone and standing position in osteoarthritis by topical phytotheraputic treatment irrespective of age and sex. Int J Phytomedicine 7: 290301.

14. Belcaro G, Dugall M, Luzzi R, Hosoi M, Ledda A, et al. (2018) Phytoproflex ${ }^{\circledR \text { : }}$ supplementary management of osteoarthrosis: a supplement registry.
Minerva Med 109: 88-94.

15. Wang Q, Pan X, Wong HH, Wagner CA, Lahey LJ, et al. (2014) Oral and topical boswellic acid attenuates mouse osteoarthritis. Osteoarthritis Cartilage 22: 128-132.

16. Ramadan G, Al-Kahtani MA, El-Sayed WM (2010) Anti-inflammatory and anti-oxidant properties of Curcuma longa (turmeric) versus Zingiber officinale (ginger) rhizomes in rat adjuvant-induced arthritis. Inflammation 34: 291-301.

17. Al-Bahtiti NH (2015) A study of preservative effects of sesame oil (Sesamum indicum L.) on mashed potatoes. Int J Sci Res Innovative Technol 2: 6-10.

18. Berit D, Ingrid W, Anders B (1992) Effect of active hand exercise and wax bath treatment in rheumatoid arthritis patients. Arthritis Care Res 5: 87-92.

19. Shah U (2011) Cissus quadrangularis L.: phytochemicals, traditional uses and pharmacological activities - a review. Int J Pharm Pharm Sci 3: 41-44.

20. Souza JS, Machado LL, Pessoa OD, Braz-Filho R, Overk CR, et al. (2005) Pyrrolizidine alkaloids from Heliotropium indicum. J Braz Chem Soc 16 : 1410-1414

21. Kontogianni VG, Tomic G, Nikolic I, Nerantzaki AA, Sayyad N, et al. (2013) Phytochemical profile of Rosmarinus officinals and Salvia officinalis extracts and correlation to their antioxidant and anti-proliferative activity. Food Chem 136: 120-129.

22. Singh S, Singh S, Singh AP (2013) Phytochemical investigation of different plants parts of Calotropis gigantea. Int J Sci Res Publ 3: 1-3.

23. Singh S, Singh S, Mishra RM, Shrivastava MP (2014) Preliminary phytochemical screening of Calotropis gigantea leaf. Int J Sci Res Publ 4: 1-3.

24. Ganguly A (2017) Diagnosis, prevention \& phytotherapy for osteoarthritic disorders: pain diagnostic parameters and prevention of OADs. Scholars press, UK, pp: 17-33.

25. Ganguly A (2015) Degenerative changes in lumber-region occur simultaneously with bilateral-osteoarthritic changes in knee-joints and vice-versa: normalization with topical application of phytoconstituents by specialized techniques involving possible cartilage- regeneration. Int J Recent Sci Res 6: 6331-6346. 